\title{
Electroencephalographic and evoked potential correlates of reaction time and visual discrimination performance*
}

\author{
CHARLES J. MORRIS \\ Denison University, Granville, Ohio 43023
}

Electroencephalographic (EEG) and evoked cortical potential (ECP) measures of arousal were found to be related to level of performance on a reaction time and visual discrimination task. ECP measures were more strongly associated with performance on both tasks than any of the EEG measures. However, the very weak association between arousal measures and visual discrimination performance suggested that arousal plays a limited role in the control of complex decision processes.

The view that $t h e$ electroencephalogram (EEG) may provide a reliable index of the excitability level of the ascending reticular activating system (ARAS) was first advanced by Lindsley (1951, 1952). More recently, the evoked cortical potential (ECP) has also been proposed as a measure of ARAS "arousal" (Haider, Spong, \& Lindsley, 1964). Since electrical stimulation of the reticular formation is known to facilitate performance on sensorimotor tasks (Fuster, 1958), one would predict, on the basis of Lindsley's theory, that the EEG and ECP should be related to level of performance on these same tasks. Although some contradictory evidence exists, a number of investigators have reported findings which support Lindsley's theory (e.g., Donchin \& Lindsley, 1966; Dustman, Boswell, \& Porter, 1962; Haider et al, 1964; Morrell \& Morrell, 1966). The present investigation was designed to analyze further the relationship between the EEG and ECP and performance by comparing the relative effectiveness of these measures in predicting level of performance on two sensorimotor tasks differing in complexity-reaction time and visual discrimination.

SUBJECTS AND APPARATUS

The Ss were 16 undergraduate and graduate students, none of whom were known to suffer from neurological damage or were under medication at the time of the experiment.

An Offner Type $R$ dynograph was used to amplify and record S's brain potentials, which were stored simultaneously on an Ampex FR 1100 tape recorder for subsequent electronic analysis. Grass E5S silver cup electrodes were placed over the right occipital lobe and the vertex. All recordings were made between these two electrode sites, which corresponded to $\mathrm{O}_{2}$ and $\mathrm{C}_{2}$, respectively, of the international "10-20" system.
Visual stimuli, produced by a Grass Model PS-2 photostimulator, were used in both the reaction time and visual discrimination tasks. Each stimulus consisted of a 10-microsec flash, delivered through a translucent screen positioned $1 \mathrm{~m}$ from the S's eyes. Rate of flash presentation and intensity changes (visua) discrimination) were controlled by a tape programmer.

The $\mathrm{S}$ signaled his response on a key placed below and in contact with his right index finger. Care was taken to insure that $S$ maintained the same response style throughout the session (reaction time).

\section{PROCEDURE}

Simple Reaction Time

Following a practice session in which $S$ was acquainted with both tasks, the experiment proper was begun. In the simple reaction time (SRT) task (Session 1), $S$ was instructed to press the response key as rapidly as possible each time the light flashed. Flashes were presented on a VI 10-sec schedule. A total of 200 flashes were presented during the session.

\section{Visual Discrimination}

The visual discrimination (VD) task (Session 2) involved the detection of an increase in the brightness (critical stimulus) of visual flashes presented every 4 sec. Critical stimuli were delivered on a VI 20-sec schedule. Preliminary data revealed that Ss tended to make a large number of "false alarms" on the task; thus, all Ss were subsequently instructed to press the key only if they were absolutely certain that a critical stimulus had occurred. A total of 150 critical stimuli were presented to each S.

\section{DATA ANALYSIS}

Data Selection

The SRT data were categorized into fast (upper quartile) and slow (lower quartile) scores for each $S$. Since scores increased in latency during the session for most Ss, thus creating the possibility of differential habituation effects on the EEG and ECP under the two conditions, pairs of fast and slow responses which occurred within five trials of each other were selected for analysis. This procedure resulted in an average of 39 trials per $S$ for each performance level (fast and slow) on the SRT task.

Correct and incorrect (omissions) responses on the VD task were selected in a similar fashion. An average of 40 trials per $S$ for each performance level on the VD task were available for analysis.

EEG Measures

The EEG analysis consisted of a wavelength distribution analysis and a voltage analysis of the EEG during the 2 -sec epoch prior to each selected stimulus presentation in the SRT task and prior to each selected critical stimulus in the VD task. The wavelength analysis computed the number of waves occurring in the alpha $(8-13 \mathrm{~Hz})$, beta $(14-30 \mathrm{~Hz})$, theta $(4-7 \mathrm{~Hz})$, and delta $(1-3 \mathrm{~Hz})$ frequency bands in each 2-sec epoch. The voltage analysis computed the rectified voltage across the same 2 -sec epochs.

Evoked Potential Measures

Each ECP selected for analysis was electronically analyzed at 8-msec sampling intervals for a total of 500 msec following the onset of the flashes in the SRT task and the onset of the critical stimulus in the VD task. The ECPs elicited during each performance level of each task were then summed and averaged. This technique provided a clear separation of the ECP from the background EEG activity for all Ss. The amplitude and latency of each component of the averaged ECP (see Fig. 1) were then computed for each $S$ for each performance level on the SRT and VD tasks.

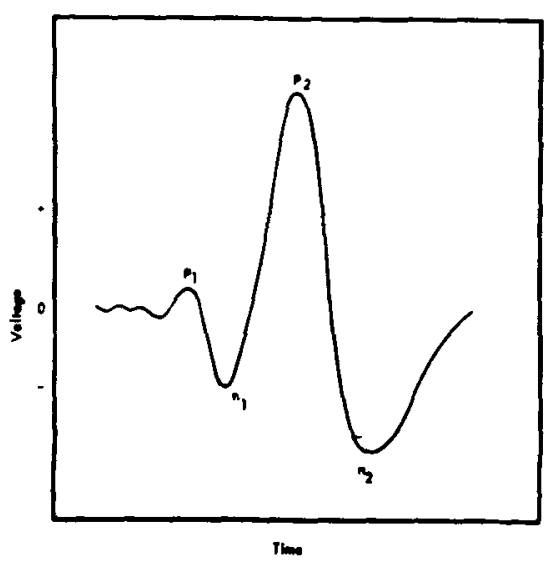

Fig. 1. The components of a typical averaged evoked potential. 

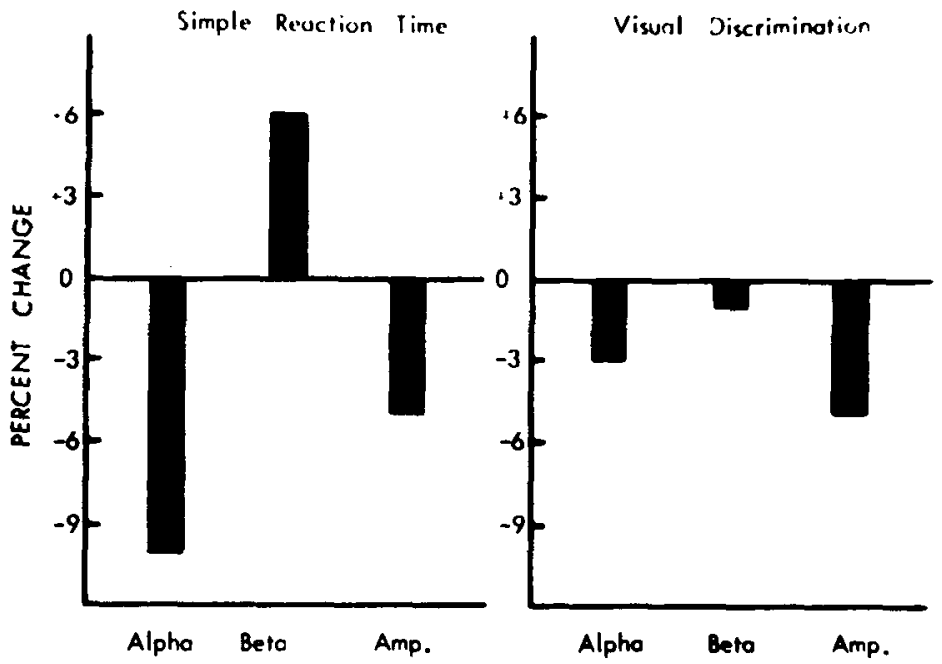

Fig. 2. Percent change in alpha and beta incidence and average EEG amplitude in the 2-sec epoch prior to fast vs slow SRT performance and correct vs incorrect (omissions) VD performance.

Statistical Evaluation

$T$ tests for paired observations were used to test for EEG and ECP differences between fast and slow performance on the SRT task and correct and incorrect (omissions) responses on the VD task. Separate analyses were computed for each type of EEG activity (alpha, beta, theta, and delta incidence), EEG voltage, and the amplitude and latency of each component of the averaged ECP (see Fig. 1). In addition, "strength of statistical association"' indices (Hays, 1963) were determined for each analysis. This measure provided an estimate of the extent to which each measure was associated with level of performance in the SRT and VD tasks. RESULTS

EEG Analysis

Simple reaction time. Compared with the EEG prior to slow SRT scores, the EEG prior to fast SRT performance was characterized by significantly reduced alpha incidence $(t=3.53, \quad p<.001)$, increased beta incidence $(t=3.10, p<.01)$, and reduced amplitude $(t=2.58, p<.05)$. These differences are shown graphically in the left-hand panel of Fig. 2 in terms of the percent change in alpha and beta incidence and average amplitude per wave in the 2 -sec epoch prior to fast as compared with slow SRT performance.

Visual discrimination. Neither alpha nor beta incidence were found to be significantly different prior to correct and incorrect (omissions) VD performance. Average amplitude, however, was found to be reliably smaller prior to correct detections of the critical stimuli $(t=2.13, p<.05)$. The right-hand panel of Fig. 2 shows the percent change in these measures during correct as compared to incorrect performance of the VD task.

Evoked Potential Analysis

Simple reaction time. Significantly larger amplitudes of the $n_{2}$ and $p_{2}$ components of the ECP were found for fast SRT performance $(t=4.56$ for $n_{2}$ and $t=3.74$ for $p_{2}, p<.001$ in both cases). No differences were found between $n_{1}$ and $p_{1}$ under the two conditions and latencies of the various components of the ECP were remarkably stable under all conditions. Average amplitude and latency of each component of the ECP during fast and slow SRT performance are plotted in the left-hand portion of Fig. 3 .
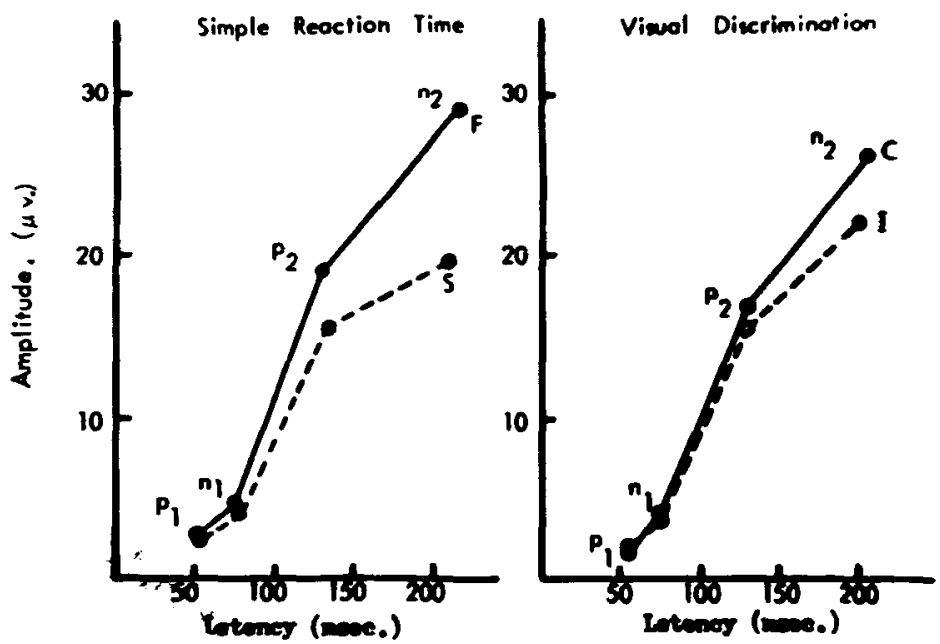

Fig. 3. Mean amplitude and latency of each peak of the ECP elicited during fast (F) and slow (S) SRT performance and correct (C) and incorrect (I) VD performance. 
Table 1

\begin{tabular}{lccccccc}
\hline & \multicolumn{2}{c}{ ECP } & & & \multicolumn{2}{c}{ EEG } \\
\cline { 2 - 4 } & $\mathrm{p}_{2}$ & $\mathrm{n}_{2}$ & & & Alpha & Beta & Amplitude \\
\hline Simple Reaction Time & .14 & .45 & & .06 & .05 & .03 \\
Visual Discrimination & - & .09 & & - & - & .02 \\
\hline
\end{tabular}

The late components of the ECP, in particular $n_{2}$, were also found to be more strongly related to level of performance than any of the EEG measures. It should be noted that combined frequency and amplitude EEG measures did not substantially raise (no more than two percentage points) the strength of statistical association values reported above. Moreover, response-produced effects on the ECP are an unlikely reason for the superiority of the ECP in predicting performance. Ss whose reaction time scores exceeded the completion of the ECP showed the same relationship between the ECP and reaction time as those $S$ s whose responses overlapped with the occurrence of the ECP. Secondly, one would expect the latency of the various components of the ECP to be modified by response-produced effects, since such effects would tend to prolong the particular phase of the potential with which they overlapped. As noted above, however, latency scores were constant under all conditions of the experiment. Thirdly, response-produced effects should have influenced the waveform of the ECP in those cases in which S's response overlapped with the occurrence of the potential. However, the idiosyncratic fluctuations of the ECP characteristic of a given $S$ were found under all conditions of the experiment and occurred invariably with the same latency.

Finally, the ability of the EEG and ECP to predict performance appears to be restricted to relatively simple tasks, in particular to those tasks which do not require decision processes. This finding is not altogether surprising, since nonsensory factors are known to have profound effects upon discrimination performance (Swets, Tanner, \& Birdsall, 1964). It does, however, suggest that arousal factors may play a limited role in the control of complex decision processes.

\section{REFERENCES}

DONCHIN, E., \& LINDSLEY, D. B Average evoked potentials and reaction times to visual stimuli Electroencephalography \& Clinical Neurophysiology, 1966, 20, 217-223.

DUSTMAN, R. E. BOSWELL, R. S., \& PORTER, P. B. Beta brain waves as an index of alertness. Science, 1962, 137, $533-534$.

FUSTER, J. M. Effects of stimulation of brain stem on tachistoscopic perception. Science, 1958, 127,150 .

HAIDER, M., SPONG, P.. \& LINDSLEY, D.

B. Attention, vigilance, and cortical evoked potentials in humans. Science. $1964,145,180-182$.

HAYS, W. L. Statistics for psychologists. New York: Holt, Rinehart, \& Winston, 1963.

LINDSLEY, D. B. Emotion. In S. S. Stevens (Ed.), Handbook of experimental psychology. New York: Wiley, 1951. Pp. 473-516.

LINDSLEY, D. B. Psychological phenomena and the electroencephalogram. Electroencephalography \& Clinical Neurophy siology, 1952, 4, 443-456.
Following overtraining on a two-choice visual discrimination, Mandler (1968) found that changing the positive stimulus $\left(S^{+}\right)$disrupted subsequent performance more than changing the negative stimulus $(S-)$. Following mastery training only, there was a small but nonsignificantly greater disruption when $\mathrm{S}-$ was changed. It was concluded that one of the effects of overtraining was an increase in control by $S^{+}$and a decrease in control by $\mathrm{S}-$ over choice behavior. Since reacquisition following overtraining proceeds very rapidly, regardless of which stimulus is changed, it seems likely that real differences in positive and negative stimulus control might be partially masked by ceiling effects. Furthermore, when the testing procedure involves the same stimuli used in acquisition, it is not possible to determine whether stimulus control is specific to the stimuli used in acquisition or whether it is more general in nature. It was decided, therefore, to use a transfer test which would produce more errors as well as involve new visual stimuli, yet which

* This work was supported in part by Research Grant MH $\mathbf{1 6 8 5 0}$ from the National Institute of Mental Health.
MORRELL, L. K., \& MORRELL, F. Evoked potentials and reaction times: $A$ study of intra-individual variability. Electroencephalography \& Clinical Neurophysiology, 1966, 20, 567-575.

SWETS, J. A., TANNER, W. P., \& BIRDSALL, T. G. Decision processes in perception. In J. A. Swets (Ed.), Signal detection and recognition by human observers. New York: Wiley, 1964. Pp. 3-57.

\section{NOTE}

1. This report is based upon a dissertation submitted in partial fulfillment of the requirements for the $\mathrm{PhD}$ degree, University of Missouri, 1967. Part of the research was supported by a NASA grant to Robert $S$. Daniel, to whom the author is indebted for his guidance during the course of the investigation and during his entire graduate career.

\title{
Multiple stimulus discrimination learning, II. Effects of prior training*
}

\author{
JEAN M. MANDLER \\ University of California at San Diego, La Jolla, Calif. 92037
}

Performance on multiple-stimulus visual discriminations improved as a function of amount of prior training on another visual discrimination. Performance on the multiple-stimulus tasks, however, indicated no changes in positive and negative stimulus control as prior training was increased from criterion performance to include overtraining. It was concluded that the greater $S+$ control following overtraining found in previous data may not be generalized stimulus control but specific to the stimuli used in acquisition.

would still make it possible to detect differences in positive and negative stimulus control.

One way to test the relative control of $S^{+}$and $S-$ is to vary the number of positive and negative stimuli used in a given problem. Mandler (1970a) found that a discrimination task in which there was one S- and three positive stimuli was more difficult than a standard $\mathrm{S}+\mathrm{S}-$ task but easier than a task with one $\mathrm{S}+$ and three negative stimuli. This type of discrimination problem weights the relative importance of $S+$ and $S-$ in acquisition and should be sensitive to biases in positive and negative stimulus control established by prior training. It was decided to study acquisition of such multiple-stimulus discriminations following mastery or overtraining on a standard $\mathrm{S}+\mathrm{S}-$ discrimination. If stimulus control is changing during the course of original acquisition from relatively equal control at mastery to greater control by $\mathbf{S}+$ following overtraining, these differences should affect the acquisition of a task in which S+ and S- are differentially weighted. If $\mathrm{S}+$ is controlling choice behavior more than $\mathrm{S}-$, a task with constant $\mathrm{S}+$ /multiple $\mathrm{S}-$ should be easier than a task with a multiple $\mathrm{S}+$ /constant S-. The results on the multiple task should be the opposite if 